\title{
A reappraisal of thrombolysis in primary deep vein thrombosis of upper extremities: Be conservative
}

\author{
George Geroulakos ${ }^{1}$, Alexei SvetlikovD \\ ${ }^{1}$ Department of Vascular Surgery, Attikon University Hospital, Athens, Greece \\ ${ }^{2}$ Department of Vascular and Endovascular Surgery, Stroke and MI prevention Center, Thrombosis and Hemostasis Center, St-Petersburg, Russian Federation
}

\begin{abstract}
Thrombolysis is increasingly used in academic centres as first line management in patients who present within the first two weeks of the index event and upper extremity primary deep vein thrombosis (UEDVT). The purpose of this report is to establish whether this approach is evidence based. A review of the recent literature showed that thrombolysis should be offered to a small number of young and active patients with severe acute symptoms, who are very concerned about developing post-thrombotic syndrome. The majority of the patients can still be treated with anticoagulation alone as there is only limited evidence on the merits of thrombolysis for the initial management of patients with UEDVT.
\end{abstract}

Keywords: Primary upper extremity deep vein thrombosis, thrombolysis.

The Acute Venous Thrombosis: Thrombus Removal with Adjunctive Catheter-Directed Thrombolysis (ATTRACT) trial has drawn closer scrutiny and discussion on the merits of thrombolysis in the management of acute iliofemoral deep vein thrombosis (DVT).

In his editorial, Vedantham succinctly summarized the findings of the ATTRACT trial investigating pharmacomechanical catheter-directed thrombolysis (PCDT) in patients with proximal DVT. The results showed that PCDT did not prevent post-thrombotic syndrome (PTS) for two years, increased major bleeding, did not influence health-related quality of life (QoL) or recurrent venous thromboembolism, and improved leg swelling and pain over 30 days. ${ }^{[1]}$ The question is whether a similar response could be expected on patients with primary upper extremity deep vein thrombosis (UEDVT) initially treated with thrombolysis in addition to anticoagulation compared to patients treated with anticoagulation alone. There are similarities in the etiology of primary iliofemoral and subclavian vein thrombosis as both conditions are considered overall the result of external compression. In case of iliofemoral venous thrombosis, significant stenosis is treated with venous stenting, while stenosis uncovered by thrombolysis in UEDVT is treated by first rib resection. However, there are no randomizedcontrolled trials studying UEDVT and, therefore, a head-to-head comparison of the available evidence with the ATTRACT trial is not possible. A recent Cochrane systematic review has demonstrated that there is currently limited evidence from which to draw conclusions on the benefits or harms of thrombolysis in the treatment of individuals with acute UEDVT. ${ }^{[2]}$ Despite this conclusion, a series of recent articles and reviews have suggested that, in acute cases of UEDVT, immediate thrombolysis should be performed..$^{[3-6]}$ Therefore, it still remains a controversial issue and a reappraisal of the place of thrombolysis for the management of patients with UEDVT is clinically relevant. Now, let us take a closer look at available evidence.

Received: March 02, 2020 Accepted: March 05, 2020 Published online: March 23, 2020

Correspondence: George Geroulakos, MD. Department of Vascular Surgery, Attikon University Hospital, 1 Rimini str, Chaidari, 12462 Athens, Greece. e-mail: ggeroulakos@med.uoa.gr 
In a case-control study, 45 consecutive patients who were treated for primary UEDVT received either oral anticoagulant therapy alone $(n=14$, Group 1), thrombolysis followed by anticoagulant therapy $(n=14$, Group 2), or thrombolysis, transaxillary first rib resection and anticoagulant therapy (n=17, Group 3). ${ }^{[7]}$ The study endpoints were persisting symptoms and QoL. The mean follow-up was $57 \pm 46$ (range, 2 to 176) months. Patients in Groups 2 and 3 had significantly less pain, swelling, and fatigue in the affected limb at six weeks. However, there was no significant difference in pain $(p=0.90)$, swelling $(p=0.58)$, fatigue $(\mathrm{p}=0.61)$, functional impairment $(\mathrm{p}=0.61)$, recurrence $(p=0.10)$ or $\mathrm{QoL}(\mathrm{p}=0.25)$ among the groups at the end of follow-up. Treatment strategy was not predictive of $\mathrm{QLL}$ ( $\mathrm{p}=0.91$, analysis of variance). No significant differences in the long-term symptoms or QoL between those with successful and unsuccessful thrombolysis were observed. The author concluded that thrombolysis with or without first rib resection did not appear to contribute to sustained symptom reduction and QoL improvement. In an earlier study, Héron et al. ${ }^{[8]}$ reported no significant association between the occurrence of PTS and ultrasonographic findings after conventionally treated spontaneous UEDVT.

In another interesting retrospective analysis of 103 patients who had 110 first rib resections, 45 subclavian veins underwent thrombolysis with or without venoplasty prior to first rib resection and were compared to 65 subclavian veins on preoperative anticoagulation alone. ${ }^{[9]}$ Of the veins treated with endovascular method, $91 \%$ were patent with symptomatic relief. In the group of veins treated with anticoagulation alone prior to first rib resection at 16 months of follow-up, 91\% were patent with symptomatic relief. The authors concluded that preoperative thrombolysis provided no benefit over simple anticoagulation. To the best of our knowledge, there is no other recent institutional case series available comparing thrombolysis with anticoagulation in the initial management of acute primary UEDVT.

On the other hand, thrombolysis may be associated with harmful effects. In a series of 30 patients with UEDVT, major bleeding was recorded in three patients (10\%). ${ }^{[10]}$ In another series of 16 patients who underwent ultrasound-accelerated catheterdirected thrombolysis for UEDVT, two patients had gastrointestinal bleeding. ${ }^{[4]}$

Some of the results of the aforementioned studies are very similar to the findings of the ATTRACT trial. Due to the limited data from studies regarding the management of UEDVT, recommendations on selected topics are usually from extrapolation of studies from DVT in the lower extremities. It has been shown that, during the course of anticoagulation, patients with UEDVT have a similar outcome with those with lower extremity DVT. ${ }^{[1]]}$ It seems that thrombolysis not only in proximal lower extremity DVT, but also in UEDVT should be used with caution in carefully selected patients until Level I evidence indicates the validity of this approach in the management of the full spectrum of patients with UEDVT. This cautious approach is also adopted by the 2012 American College of Chest Physicians guideline that has averted clinicians from performing routine thrombolysis and should be restricted to very few cases, if any. ${ }^{[12]}$ Our reappraisal of the most recent literature suggests that this guideline is still valid.

\section{Declaration of conflicting interests}

The authors declared no conflicts of interest with respect to the authorship and/or publication of this article.

\section{Funding}

The authors received no financial support for the research and/or authorship of this article.

\section{REFERENCES}

1. Vedantham S. The Attract Trial: A Step Forward for Evidence Based DVT Care. Eur J Vasc Endovasc Surg 2018;56:320-1.

2. Feinberg J, Nielsen EE, Jakobsen JC. Thrombolysis for acute upper extremity deep vein thrombosis. Cochrane Database Syst Rev 2017;12:CD012175.

3. Keir G, Marshall MB. Management Strategy for Patients With Chronic Subclavian Vein Thrombosis. Ann Thorac Surg 2017;103:672-5.

4. Ozcinar E, Yaman ND, Cakici M, Baran C, Inan MB, Durdu S, et al. Pharmacomechanical thrombectomy of upper extremity deep vein thrombosis. Int Angiol 2017;36:275-80.

5. Mahmoud O, Vikatmaa P, Räsänen J, Peltola E, Sihvo E, Vikatmaa L, et al. Catheter-Directed Thrombolysis Versus Pharmacomechanical Thrombectomy for Upper Extremity Deep Venous Thrombosis: A Cost-Effectiveness Analysis. Ann Vasc Surg 2018;51:246-53.

6. Carlon TA, Sudheendra D. Interventional therapy for upper extremity deep vein thrombosis. Semin Intervent Radiol 2017;34:54-60.

7. Bosma J, Vahl AC, Coveliers HM, Rauwerda JA, Wisselink W. Primary subclavian vein thrombosis and its long-term effect on quality of life. Vascular 2011;19:327-32.

8. Héron E, Lozinguez O, Emmerich J, Laurian C, Fiessinger JN. Long-term sequelae of spontaneous axillary-subclavian venous thrombosis. Ann Intern Med 1999;131:510-3. 
9. Guzzo JL, Chang K, Demos J, Black JH, Freischlag JA. Preoperative thrombolysis and venoplasty affords no benefit in patency following first rib resection and scalenectomy for subacute and chronic subclavian vein thrombosis. J Vasc Surg 2010;52:658-62.

10. Vik A, Holme PA, Singh K, Dorenberg E, Nordhus $\mathrm{KC}$, Kumar S, et al. Catheter-directed thrombolysis for treatment of deep venous thrombosis in the upper extremities. Cardiovasc Intervent Radiol 2009;32:980-7.

11. Cote LP, Greenberg S, Caprini JA, Tafur A, Choi C, Muñoz
FJ, et al. Comparisons Between Upper and Lower Extremity Deep Vein Thrombosis: A Review of the RIETE Registry. Clin Appl Thromb Hemost 2017;23:748-754.

12. Guyatt GH, Akl EA, Crowther M, Gutterman DD, Schuünemann HJ; American College of Chest Physicians Antithrombotic Therapy and Prevention of Thrombosis Panel. Executive summary: Antithrombotic Therapy and Prevention of Thrombosis, 9th ed: American College of Chest Physicians Evidence-Based Clinical Practice Guidelines. Chest 2012;141(2 Suppl):7S-47S. 